\title{
Des considérations sur la lexico- graphie et la linguistique au Gabon*
}

\author{
Guy-Modeste Ekwa Ebanéga (13751735@sun.ac.za) et \\ Fatima Tomba Moussavou(13751743@sun.ac.za), Département d'afrikaans \\ et de néerlandais, Université de Stellenbosch, Stellenbosch, République \\ d'Afrique du Sud
}

\begin{abstract}
Résumé: Le Gabon est un pays à diversité linguistique. La constitution gabonaise reconnaît le français comme l'unique langue officielle. En outre, elle s'efforce de promouvoir et de protéger les langues nationales. La lexicographie, discipline de la production des dictionnaires, et la linguistique, l'étude scientifique du langage, ont toutes les deux un rôle à jouer dans la promotion et la protection des langues nationales. L'objectif de cet article est de montrer l'importance d'une collaboration entre lexicographes et linguistes pour un développement effectif des langues du Gabon.

Pour rendre compte de la nécessité de cette collaboration entre les lexicographes et les linguistes au Gabon, cet article commence par la présentation de la situation linguistique du Gabon. Ensuite des aspects tels que les contributions des deux disciplines de la linguistique et de la lexicographie au développement des langues gabonaises, la collaboration entre les linguistes et les lexicographes et la question des unités lexicographiques gabonaises seront discutés.
\end{abstract}

Mots-clés: LEXICOGRAPHIE, LINGUISTIQUE, COLLABORATION, LEXICOGRAPHES, LINGUISTES, SCIENCES INTERDISCIPLINAIRES, LANGUES GABONAISES, DICTIONNAIRE, LANGUE, CORPUS, UNITÉS LEXICOGRAPHIQUES, DÉVELOPPEMENT

\begin{abstract}
Considerations on Lexicography and Linguistics in Gabon. Gabon is a country with a linguistic diversity. The Gabonese constitution recognizes French as the only official language. Moreover, it endeavours to promote and protect the national languages. Lexicography, the discipline for the producing of dictionaries, and linguistics, the scientific study of language, both have a role to play in the promotion and protection of the national languages.

To account for the necessity of this collaboration between lexicographers and linguists in Gabon, this article starts by presenting the linguistic situation in Gabon. Then aspects such as the contribution of the two disciplines of linguistics and lexicography to the development of the Gabonese languages, the collaboration between linguists and lexicographers and the question of Gabonese lexicographic units will be discussed.
\end{abstract}

Keywords: LEXICOGRAPHY, LINGUISTICS, COLLABORATION, LEXICOGRAPHERS, LINGUISTS, INTERDISCIPLINARY SCIENCES, GABONESE LANGUAGES, DICTIONARY, LANGUAGE, CORPUS, LEXICOGRAPHIC UNITS, DEVELOPMENT

* Cet article est une version adaptée de la communication présentée à la Neuvième Conférence Internationale Annuelle de l'Association Africaine pour la Lexicographie (AFRILEX), organisée par le Groupe de Recherche en Langues et Cultures Orales (GRELACO), Université Omar Bongo, Libreville, Gabon, 18-23 juillet 2004. 


\section{Introduction}

Le Gabon, comme la majorité des pays africains, est un pays à diversité linguistique. À côté des langues locales, coexistent les langues parlées par les différentes communautés linguistiques d'immigrants ou de migrants de différents pays. La majorité des langues gabonaises est bantoue. Le français jouit d'un statut particulier en tant que langue introduite par la colonisation; il jouit également d'un statut priviligié parmi toutes les langues présentes au Gabon. La constitution gabonaise reconnaît le français comme l'unique langue officielle. En outre, elle s'efforce de promouvoir et de protéger les langues nationales. La lexicographie, discipline de la production des dictionnaires, et la linguistique, l'étude scientifique de la langue, ont toutes les deux un rôle à jouer dans la promotion et la protection des langues nationales. L'objectif de cet article est de montrer l'importance d'une collaboration entre lexicographes et linguistes pour un développement effectif des langues du Gabon.

Pour rendre compte de la nécessité de cette collaboration entre les lexicographes et les linguistes au Gabon, notre propos commencera par la présentation de la situation linguistique au Gabon. Des aspects tels que les contributions des deux disciplines linguistiques et lexicographiques au développement des langues gabonaises, la collaboration entre les linguistes et les lexicographes et la question des unités lexicographiques gabonaises seront également discutés.

\section{Situation linguistique}

Le Gabon est un pays multilingue avec plus de 40 langues gabonaises; en outre le français est la langue officielle (cf. Grimes 1996: 1). Il est également la langue véhiculaire et la langue d'ascension sociale (cf. Mavoungou 2002: 230), langue de la communication individualisée et institutionalisée1 (cf. Mba-Nkoghe 1991: 20-23), ainsi que la langue d'immigrants africains syro-libanais d'introduction récente. La majorité des langues gabonaises est bantoue selon les travaux de Guthrie (1953: 55-73), Doke et Cole (1963: 63-76), Jacquot (1978) et Kwenzi-Mikala (1990). Parmi les langues gabonaises, on peut ajouter celles parlées par les pygmées; elles ne sont pas très bien connues. Les langues gabonaises peuvent être divisées en dix groupes de parlers ou unités-langues. Une unité-langue est l'ensemble de différents parlers, tous mutuellement compréhensibles.

Bon nombre de recherches ont été faites sur les classifications en langues gabonaises; d'après les données dont nous disposons, on peut citer les travaux de Grimes (1996), Jacquot (1978), Raponda-Walker (1960) et Guthrie (1953). La classification la plus récente est celle de Kwenzi-Mikala (1987, 1998). Selon Kwenzi-Mikala, on relève dix groupes de parlers ou unités-langues à savoir les groupes mazuna, myene, mekana-menaa, mekona-mangote, membe (ou okandetsogo), merye, metye, membere, makena et baka. Ces groupes de langues sont établis sur le critère d'intercompréhension mutuelle et la formule de conversation «je dis que» (à l'exception du groupe baka). 
Malgré l'article de la constitution qui reconnaît le français comme langue officielle, en même temps s'efforce de promouvoir et protéger les langues nationales, aucune langue gabonaise n'a le statut de langue officielle. Emejulu et Nzang-Bié (1999) ont utilisé la grille théorique de Fishman (1991) pour évaluer le niveau de vie et la dynamique des langues nationales. Il resort de cette étude qu'au mieux toutes ces langues nationales se classent invariablement des stades 5 à 8 selon leur degré de valence sociale.

Cependant toutes ces langues gabonaises ne sont ni développées, ni standardisées, ni codifiées, ni utilisées dans aucune structure officielle sauf pour quelques unes (et ce d'une manière $a d$ hoc) à la Radio Nationale ou Provinciale selon les besoins politiques. De récents travaux sur la vitalité des langues gabonaises montrent d'ailleurs des résultats très alarmants (Idiata 2003a). Parmi ces langues, certaines d'entre elles dont les seuls locuteurs sont quelques vieillards dans les villages, sont simplement menacées d'extinction (cf. Emejulu 2001: 39).

Quand le gouvernement a pris conscience de l'importance des langues nationales dans le développement du pays, il a commencé à les promouvoir. À cet effet, un Séminaire sur les États Généraux de l'Éducation et de la Formation s'est réuni du 12 au 13 décembre 1983. L'une des recommandations du séminaire était l'introduction des langues d'héritage culturel dans le système éducatif et national. Cependant, aucune mesure concrète ne découle de cette recommandation. Quatorze ans plus tard, cette recommandation fut encore introduite pendant la Table Ronde sur les Recherches Linguistiques et l'Enseignement des Langues au Gabon qui s'est tenue du 9 au 11 décembre 1997.

Recemment en avril 1997, le Séminaire sur la Standardisation de l'Orthographe des Langues Gabonaises était organisé par le Ministre de l'Éducation Nationale.

Les langues nationales sont utilisées comme médium d'instruction dans le programme littéraire pour adultes et à la télévision. Une section sur les langues nationales a vu le jour pendant l'année académique 1998-1999 à l'École Normale Supérieure (ENS) de Libreville. Malheureusement, ce projet n'aboutit toujours pas. Trois raisons expliquent cette situation:

(a) Les gens pensent que la promotion et le développement des langues sont l'affaire des linguistes.

(b) Les linguistes ne sont pas nombreux au Gabon; on y dénombre environ une quinzaine.

(c) Le travail des linguistes n'est pas accessible au public puisqu'il est très technique. Ils se perdent aussi dans des descriptions phonologiques.

Pour cela, il nous faut faire une projection de l'avenir, c'est-à-dire compter sur le soutien de quelques lexicographes gabonais formés à l'Université de Stellenbosch (Afrique du Sud) pour que l'étude des langues gabonaises soit effective. 


\section{Linguistique, lexicographie et développement des langues gabonaises}

La lexicographie et la linguistique contribuent au dévéloppement des langues gabonaises. La linguistique traite les classifications des langues et des descriptions linguistiques. Sur le domaine des classifications des langues gabonaises, nous pouvons mentionner les recherches de Kwenzi-Mikala (1990) qui a énumeré les langues gabonaises dans 62 parlers, catégorisés dans dix groupes de langues selon leur intelligibilité mutuelle.

Quant aux descriptions linguistiques, elles regroupent les domaines de la phonétique, la phonologie et la morphologie. Les descriptions du domaine de la syntaxe, de la sémantique, de la psycholinguistique et de la sociolinguistique sont rares et sont limitées à quelques langues (Idiata 2003: 37). Les descriptions linguistiques sont entreprises au Département des Sciences du Langage de l'Université Omar Bongo, Libreville, créé en 1994. L'étude des langues gabonaises demeure un vaste champ d'investigation à explorer.

Les contributions lexicographiques commencent vers les années 1800 par les missionnaires et les administrateurs coloniaux. Ces derniers, dans leur souci d'évangélisation et de communication avec les populations gabonaises, ont produit des ouvrages à caractère lexicographique. Bien que perfectibles, ces travaux répondent aux critères métalexicographiques, notamment pour les méthodes utilisées pour la compilation des dictionnaires. La sémantique, la syntaxe et la pragmatique sont negligées dans ces dictionnaires. En outre, les dictionnaires compilés par les missionnaires et administrateurs coloniaux étaient des dictionnaires bilingues et étaient produits pour donner aux non-locuteurs natifs des langues gabonaises accès à la langue étrangère.

Par ailleurs, le Gabon a connu des développements importants dans le domaine lexicographique. Un séminaire sur la lexicographie bantoue tenu au Centre International de Civilisation Bantoue (CICIBA) a eu lieu en 1997 à Libreville par les membres venus de six pays africains (Cameroun, Centrafrique, Gabon, Guinée Equatoriale, Rwanda et São Tomé et Principé). Au cours de ce séminaire, le Dr. D.J. van Schalkwyk et le Dr. F.J. Lombard, éditeur et éditeur adjoint précédents du Woordeboek van die Afrikaanse Taal (WAT), ont partagé avec leurs homologues africains leur savoir sur la métalexicographie et la pratique lexicographique en Afrique et dans le monde. Un accord de coopération liant le Groupe de Recherches en Langues et Cultures Orales (GRELACO) de l'Université Omar Bongo et le Bureau du Woordeboek van die Afrikaanse Taal (WAT) fut signé le 6 décembre 1999. Toujours en 1999, deux étudiants gabonais sont venus au Bureau du WAT pour une formation continue et pour leur étude doctorale en lexicographie à l'Université de Stellenbosch (République d'Afrique du Sud) sous la direction du professeur R.H. Gouws. En 2000, trois autres étudiants sont venus en Afrique du Sud et en 2001 ils ont été suivis par cinq autres. Le Bureau du WAT leur a donné une formation pilote en lexicographie générale, en lexicographie informatique et en planification des projets lexicographiques. Cinq étudiants ont soutenu leur thèse de docteur en lexicographie. 
Trois ans plus tard s'est tenue à Libreville du 18 au 23 juillet 2004, la Neuvième Conférence Internationale de l'Association Africaine pour la Lexicographie (AFRILEX). Le sujet de cette conférence était la problématique du dictionnaire, le développement des langues et l'organisation des centres lexicographiques. Son objet était la recherche des zones de convergences entre le dictionnaire et la langue, afin de proposer une stratégie dynamique pour le développement de la lexicographie gabonaise, appelée à rendre accessibles les connaissances du monde contemporain pour la pluralité des langues et cultures du Gabon.

Il semble toutefois que le dictionnaire est très peu utilisé au Gabon. On consulte généralement un dictionnaire pour chercher le sens d'un mot ou pour vérifier son orthographe. Il ne fait pas partie des ouvrages courants à consulter, et est donc rangé dans la catégorie des ouvrages occasionnels. Les dictionnaires dans les langues gabonaises ne se trouvent pas dans les librairies, les rayons des bibliothèques générales, et dans les établissements scolaires et universitaires. Il est donc primordial qu'une culture du dictionnaire s'établisse au Gabon. On entend, par «culture du dictionnaire», accoutumer l'usager à l'emploi du dictionnaire. Au Gabon, cette culture du dictionnaire peut s'établir au moyen du système éducatif. Il est important que la culture du dictionnaire s'installe chez les enseignants, qui à leur tour transmettront leur connaissance lexicographique aux élèves et étudiants. Il incombe aux lexicographes de véhiculer cette culture.

Les structures telles que l'École Normale des Instituteurs (ENI), l'École Normale Supérieure (ENS) et l'Université Omar Bongo (UOB) sont appropriées pour transmettre des connaissances lexicographiques aux éducateurs qui, à leur tour, les inculqueront aux élèves et étudiants.

\section{Collaboration entre linguistes et lexicographes}

Il importe qu'une collaboration entre linguistes et lexicographes s'établisse au Gabon. Cette collaboration peut être effective si l'on considère le rôle joué par le dictionnaire comme miroir de la société, le réservoir du savoir linguistique et culturel. Dans le but de préserver la riche variété des langues gabonaises, les lexicographes et linguistes devraient travailler ensemble. Les premiers, les lexicographes, devraient réviser ou améliorer les dictionnaires bilingues existants ${ }^{2}$ en se penchant sur les types de dictionnaires tels les dictionnaires monolingues basés sur des descriptions linguistiques. Selon Tomba Moussavou (2002: 159$160)$, ces dictionnaires monolingues seront destinés aux usagers natifs; ils seront également destinés aux usagers qui apprennent une seconde langue. L'avènement de ces dictionnaires en langues gabonaises aura plusieurs incidences sur la standardisation, la terminologie, les recherches linguistiques ainsi que l'enseignement des dites langues. En outre, ces dictionnaires se proposeront, d'une part, d'améliorer et de compléter les carences et insuffisances aussi bien macro- que microstructurelles des dictionnaires qui ont été compilés par 
les missionnaires, et d'autre part les domaines que ces dictionnaires bilingues n'ont pas exploités. Quant aux dictionnaires bilingues déjà existants, nous sommes tout à fait du même avis que Afane Otsaga (2001: 138,157) optant pour une nouvelle approche et un modèle théorique de compilation des dictionnaires bilingues, en tenant compte du contexte spécifique dans lequel se trouve les langues gabonaises. Selon l'auteur, cette nouvelle approche devrait partir de la collection des données, au contenu même des articles du dictionnaire bilingue: la traduction, l'équivalence, les synonymes, les exemples et la forme des entrées. Quelques aspects généraux sur le dictionnaire bilingue tels que la sélection des entrées et les relations sémantiques devraient aussi être revisitées. Les deuxièmes, les linguistes, devraient orienter leurs travaux dans des descriptions sémantiques et syntaxiques, qui sont encore jusque-là inexplorées; il faut noter que les travaux de syntaxe sont assez rares et limités à quelques langues qui ont fait l'objet de recherches doctorales en morphosyntaxe, comme le isangu (groupe merye) (cf. Idiata 2003: 37).

Il existe aujourd'hui beaucoup de descriptions linguistiques sur les langues gabonaises - même si certains domaines précédemment cités restent inexplorés - depuis la création du Département des Sciences du Langage au sein de la Faculté des Lettres et Sciences Humaines (FLSH) de l'Université Omar Bongo. Ces descriptions ne sont pas accessibles au public, puisque très techniques et hermétiques, comme énoncé dans les lignes précédentes. Dans cette situation, une collaboration entre lexicographes et linguistes s'impose, pour que la connaissance de la langue soit accessible au public. En effet, les lexicographes pourraient se servir des travaux linguistiques et intégrer les aspects qui les intéressent dans les dictionnaires, sans avoir besoin eux-mêmes de refaire le travail d'analyse linguistique. En outre, le dictionnaire reste l'élément le plus approprié pour rendre effectif la connaissance de la langue. Il donne à la communauté la possibilité d'évaluer la langue: «donne moi ton dictionnaire et j'évaluerai ta langue». Le dictionnaire va développer l'essence même de la langue.

Les lexicographes et les linguistes doivent orienter leurs ressources et leurs efforts dans la compilation des corpora en langues gabonaises. Le corpus se définit comme un corps de textes écrits ou un discours transcrit qui peut servir de base à une analyse et description linguistique (Kennedy 1998: 1; Ekwa Ebanéga 2002: 188). L'objectif du corpus est de permettre aux lexicographes et linguistes de mener des recherches en tenant compte des réalités linguistiques issues du langage quotidien des usagers-cibles (Ekwa Ebanéga 2002: 190). Au Gabon, le corpus serait une importante source permettant aux chercheurs de travailler de façon permanente sur les langues gabonaises. Selon Emejulu (2001: 45), une rupture épistémologique franche s'impose pour que les études sur les langues gabonaises aient une utilité pratique et connaissent un essort que l'on attend d'elles. Pour atteindre ce but, les lexicographes et les linguistes gabonais doivent désormais orienter leurs efforts prioritairement vers le développement des corpora électroniques. Ainsi l'existence d'un tel rassemblement de textes en 
langues gabonaises fournira également aux lexicographes et linguistes un accès à des données actuellement dispersées ou non disponibles, quelque soit leurs domaines scientifiques respectifs (lexicographie, linguistique, etc.). Nous sommes d'accord avec Emejulu (2001: 40) lorsqu'il affirme que la création et l'analyse des corpora stockés dans les banques de données informatisées ont non seulement révolutionné la linguistique, mais aussi contribué à un progrès considérable de la lexicographie. Selon lui, il est alors bien évident que si la linguistique gabonaise veut être légitime, au diapason de la science du nouveau millennium, il lui faut marquer une rupture épistémologique et donner la priorité au développement des corpora informatisées pour les langues au Gabon. $\mathrm{Au}$ niveau de l'analyse de données, nombreux sont les outils qui permettent d'analyser des corpus électroniques. Un logiciel récent qui est à la fois performant et convivial est le WordSmith Tools de Mike Scott (Oxford University Press). WordSmith effectue plusieurs types de recherches à savoir:

(a) Listes de fréquence. On peut facilement obtenir des listes de fréquence de tous les mots dans le corpus. Les listes de fréquence se présentent de deux manières: par ordre alphabétique et par ordre de fréquence.

(b) Listes comparatives. Cette fonctionnalité permet de comparer automatiquement la fréquence des mots dans des corpora différents et de faire ressortir les mots, soit beaucoup plus employés, soit beaucoup moins employés dans un des corpora.

(c) Concordances. Cette fonctionnalité permet de visualiser toutes les occurrences d'un même mot en contexte et de manipuler ce même contexte.

Grâce à cette facilité d'accès aux données et aux nouvelles possibilités de traitement et de manipulation, ce corpus, mis sous forme électronique, permettra des approches diversifiées pour les recherches dans les langues gabonaises. Il sera question ici de légitimer la construction des corpora électroniques pour toutes les langues du Gabon et de présenter les outils statistiques et informatiques pour l'analyse (qualitative et quantitative) de corpus de telle sorte que les linguistes et lexicographes gabonais soient en position de commencer dans l'immédiat à tirer profit des corpora électroniques pour les rendements meilleurs (cf. Emejulu 2001: 40). Le but final d'un tel corpus est de donner une place aux langues gabonaises dans la recherche lexicographique et linguistique du 21ème siècle.

Dans le cadre des langues africaines en général et des langues gabonaises en particulier, Ekwa Ebanéga (2002: 190) a souligné l'exemple du projet ALLEX (African Language Lexicon), à présent ALRI (African Languages Research Institute). Commencé autour de 1990, le projet ALLEX est né d'un partenariat entre les chercheurs du Département des Langages et Littérature Africaine de l'Université du Zimbabwe et ceux des Départements de Lexicographie des Universités de Göteborg (Suède) et d'Oslo (Norvège), ainsi que les hommes 
d'affaires suédois et norvégiens. Le projet ALLEX a essentiellement pour objectif la mise en place d'une base de données lexicographiques pour la production à terme des dictionnaires monolingues en mashona (Mavoungou 2001: 176). Les projets du type ALLEX pour les langues gabonaises auront pour résultat le développement de celles-ci. En termes plus concrets, un projet de l'envergure de l'ALLEX pour les langues gabonaises, aura des implications dans nombre de disciplines, notamment la lexicographie et la linguistique. D'un point de vue lexicographique et linguistique, la manipulation des vastes données est susceptible de permettre les opérations suivantes:

(a) Une analyse linguistique relativement exhaustive de chaque mot du corpus,

(b) L'identification des formes macrostructurelles (lemmes) de chaque mot, et

(c) L'identification des exemples d'emploi pour chaque mot du corpus.

L'utilisation d'un corpus peut offrir au lexicographe et au linguiste tous les contextes d'apparition du mot à l'intèrieur de la phrase, ce qui facilite énormément le travail du chercheur. Par exemple, le terme yilumbu bwâla (village), compte pour 1,10\% (soit 394 occurrences) dans le corpus yilumbu (groupe merye); il est majoritairement précédé dans les phrases par le préfixe locatif de classe 17, o (0,67\%, soit 237 occurrences dans le corpus). Bwâla s'emploie dans une moindre mesure avec des pronoms possessifs utilisés avec des noms. Les données sus-mentionnées peuvent intéresser à la fois le lexicographe et le linguiste. Pour le linguiste, l'occurrence quasi régulière du connectif $o$ en combinaison avec bwâla peut permettre de prévoir la configuration syntaxique des phrases du yilumbu dans lesquelles bwâla apparaît. Pour le lexicographe, les données présentées ci-dessus donnent déjà de bonnes indications quant aux exemples appropriés pour illustrer le lemme bwâla dans le dictionnaire.

La collaboration entre les lexicographes et les linguistes s'inscrit dans le cadre du GRELACO. Ces activités qui s'étalent dans l'espace et dans le temps sont d'interêt national puisqu'elles visent la promotion et la valorisation du patrimoine linguistique du Gabon. Elles sont reunies autour de cinq programmes majeurs, à savoir:

(a) Programme d'Alphabétisation en Langue Maternelle (PALM),

(b) Programme de Développement et Standardisation des Langues (PDSL),

(c) Programme de Dictionnaire Pédagogique et Scientifique en Langues Gabonaises (PDPSL), support majeur et permanent des PALM et PDSL,

(d) Programme d'Atlas Linguistique du Gabon (PALG), et

(e) Programme d'Acquisition et Trouble du Langage (PATL). 
Dans cette politique d'une collaboration entre lexicographes et linguistes, le GRELACO est devenu membre actif de l'Association Africaine pour la Lexicographie (AFRILEX); il a signifié son désir de collaborer d'une part avec l'Aarhus École des Affaires (Danemark), et d'autre part avec l'African Language Association for Southern Africa (ALASA).

\section{Unités lexicographiques}

La question de l'établissement des unités lexicographiques est un facteur déterminant pour la collaboration entre lexicographes et linguistes au Gabon. Une unité lexicographique est une structure de recherche dont l'objectif principal est la production pratique des dictionnaires. Dans le cadre de la politique linguistique des langues africaines, le projet de type PanSALB (Pan South African Language Board) pour les langues sudafricaines a été établi par le gouvernement de l'Afrique du Sud en 1995, afin de favoriser le multilinguisme et développer les langues qui étaient autrefois marginalisées. Grâce à ce projet l'unité lexicographique pour chacune des onze langues officielles de l'Afrique du Sud, à savoir: isizulu, isixhosa, afrikaans, sepedi, anglais, setswana, sesotho, xitsonga, siswati, tshivenda et isindebele a vu le jour. Pour le cas du Gabon, un projet de type PanSALB aurait pour résultat la préservation de la diversité linguistique et l'établissement des onze unités lexicographiques telles que les unités françaises et mazuna, myene, mekana-menaa, mekona-mangote, membe, merye, metye, membere, makena, baka, et selon les dix groupes de langues préalablement cités. Celles-ci serviront d'interface pour la collaboration entre les lexicographes et les linguistes. En établissant les unités lexicographiques au Gabon, ceci aurait comme résultat la production des dictionnaires et la préservation de la diversité linguistique sous toutes ses formes variées.

La création d'une unité lexicographique est de rigueur compte tenu des besoins lexicographiques des langues gabonaises (Nyangone Assam et Mavoungou 2000: 252 et Nyangone Assam 2001: 188). Cette structure est en train de se mettre en place et sera fonctionnelle dans le cadre du GRELACO au sein de l'Université Omar Bongo (Emejulu et Nzang-Bié 1999). Le GRELACO œuvre donc dans le sens de la création des unités lexicographiques avec le concours des communautés linguistiques. Pour satisfaire les besoins en matière des dictionnaires et produits dérivés, supports indispensables pour le développement et la standardisation des langues, il est d'une nécessité absolue de créer des unites lexicographiques à travers l'ensemble du territoire gabonais, et ce en fonction des besoins spécifiques des langues et des communautés linguistiques. Selon Emejulu (2001: 50), il est plus pratique et économique d'établir les unités lexicographiques dans les localités où se trouvent les unités-langues répertoriées. Les unités lexicographiques seront installées auprès des Académies Provinciales dans d'autres provinces que la province du Haut-Ogooué dont l'unité lexicographique sera installée au sein de l'Université des Sciences et Techniques de Masuku à Franceville. L'unité lexicographique du français sera établie 
à Libreville, la capitale du Gabon. Pour le cas de l'unité lexicographique du français, elle prendra en compte la variété du français du Gabon ${ }^{3}$ (Mavoungou 2002)

Toutes ces unités lexicographiques seront animées par des agents lexicographiques formés par le GRELACO. La première tâche des unités lexicographiques sera l'identification et la planification des projets lexicographiques à court, moyen et long termes. La deuxième et principale tâche est d'assumer la responsabilité du développement des corpora des différentes langues.

\section{Conclusion}

Dans ce travail, il a été question de l'importance d'une collaboration entre lexicographes et linguistes au Gabon. L'esprit qui a animé cette contribution était d'aller à l'essentiel et de présenter la situation linguistique du Gabon, de montrer que la lexicographie et la linguistique sont deux disciplines autonomes, avec des objets d'études bien spécifiques. La lexicographie a également une vocation interdisciplinaire, elle intretient des relations avec la linguistique et bien d'autres sciences.

Parmi les contributions de la linguistique et de la lexicographie pour le développement des langues gabonaises, il faut retenir que pour la lexicographie, la pratique des dictionnaires a bien commencé par les travaux des missionnaires et les administrateurs coloniaux; pour la linguistique, les contributions traitent de la classifications des langues et des descriptions linguistiques. La collaboration des lexicographes et des linguistes est nécessaire dans la mesure où la lexicographie permet de contenir le savoir linguistique et culturel des usagers. Les lexicographes et les linguistes doivent orienter leurs ressources et leurs efforts dans des domaines jusque-là peu explorés comme la syntaxe et la sémantique. Le corpus permet également de faire un pont entre ces deux disciplines séparées, mais qui sont amenées à interagir intimement dans l'analyse des données par l'entremise des outils performants comme le WordSmith. La création des unités lexicographiques est aussi un facteur déterminant pour la collaboration entre les lexicographes et les linguistes au Gabon. Les unités lexicographiques qui seront installées dans toutes les Académies Provinciales auront comme résultat la production des dictionnaires et la préservation de la diversité linguistique sous toutes ses formes variées. Le GRELACO s'inscrit dans le cadre d'une collaboration effective de ces deux disciplines scientifiques, compte tenu de ses activités réunies autour des programmes majeurs.

\section{Notes}

1. Le français est la seule langue dont le statut est clairement défini dans la constitution gabonaise dans l'article 2 du titre 1: "La République Gabonaise adopte le français comme langue officielle." Le français est de ce fait la langue des institutions. Le français est la langue de 
l'école, la langue du savoir, la langue de l'administration, la langue des institutions juridiques et économiques et des media.

2. Selon Kavanagh (2002: 276), tous les dictionnaires sont pratiquement basés dans une certaine mesure sur un dictionnaire déjà existant.

3. Mavoungou (2002: 230) propose des différents critères métalexicographiques pour la planification et la compilation du Dictionnaire du français du Gabon. L'auteur tient compte du fait que le français, langue officielle, est bien établi et considère le truisme selon lequel une langue ne peut exister qu'avec un seul usage. Le moment est venu de se demander si le Gabon ne doit pas assumer son propre usage du français. Il s'agit donc de poser les jalons susceptibles de nous conduire "vers un dictionnaire du français du Gabon".

\section{Bibliographie}

Afane Otsaga, T. 2001. Les dictionnaires bilingues dans les langues gabonaises: Approche méthodologique. Emejulu, J.D. (Éd.). 2001: 137-159.

Doke, C.M. et D.T. Cole. 1963. Contributions to the History of Bantu Linguistics. Johannesbourg: Witwatersrand University Press.

Ekwa Ebanéga, G.-M. 2002. Le corpus lexicographique dans le développement des langues gabonaises. Emejulu, J.D. (Éd.). 2002: 188-205.

Emejulu, J.D. 2001. Lexicographie multilingue et multisectorielle au Gabon: planification, stratégie et enjeux. Emejulu, J.D. (Éd.). 2001: 38-57.

Emejulu, J.D. (Éd.). 2001. Éléments de lexicographie gabonaise. Tome I. New York: Jimacs-Hillman.

Emejulu, J.D. (Éd.). 2002. Éléments de lexicographie gabonaise. Tome II. New York: Jimacs-Hillman.

Emejulu, J.D. et Y. Nzang-Bié. 1999. Linguistic Perspectives in Gabon. Communication presentée à un colloque organisé par le Summer Institute of Linguistics, Université de Dakota du Nord, Grand Forks, États-Unis, 20 juillet 1999.

Fishman, J.A. 1991. Reversing Language Shift: Theoretical and Empirical Foundations of Assistance to Threatened Languages. Cleveland: Multilingual Matters.

Grimes, B.F. 1996 ${ }^{13}$. Ethonologue. Dallas: Summer Institute of Linguistics, Inc.

Guthrie, M. 1953. The Bantu Languages of Western Equatorial Africa. Oxford: Oxford University Press.

Idiata, D.F. 2003. Pourquoi le Gabon doit investir sur ses langues vernaculaires. Le Cap: Centre for Advanced Studies of African Society.

Idiata, D.F. 2003a. Diversité ethnolinguistique et vitalité des langues au Gabon. Bearth, T. (Éd.). 2003. African Languages in Global Society/Les langues africaines à l'heure de la mondialisation. Cologne: Koppe Verlag.

Jacquot, A. 1978. Le Gabon. Barreteau, D. (Éd.). 1978. Inventaire des études linguistiques sur les pays d'Afrique noire d'expression française et sur le Madagascar: 493-503. Paris: CILF.

Kavanagh, K.P. 2002. Adapter un dictionnaire monolingue pour un usage local. Emejulu, J.D. (Éd.). 2002: 276-290.

Kennedy, G. 1998. An Introduction to Corpus Linguistics. Londres: Longman.

Kwenzi-Mikala, J.T. 1987. Contribution à l'inventaire des parlers bantu du Gabon. Pholia. Revue du Laboratoire de Phonétique et Linguistique Africaine 2: 103-110.

Kwenzi-Mikala, J.T. 1990. Quel avenir pour les langues gabonaises? Revue Gabonaise des Sciences de l'Homme 2: 121-124. 
Kwenzi-Mikala, J.T. 1998. Parlers du Gabon: classification du 11.12.97. Raponda-Walker, A. (Éd.). 1998. Les langues du Gabon: 217. Libreville: Éditions Raponda-Walker.

Mavoungou, P.A. 2001. La mondialisation et la lexicographie trilingue ou plurilingue au Gabon. Emejulu, J.D. (Éd.). 2001: 160-183.

Mavoungou, P.A. 2002. Vers un dictionnaire du français du Gabon. Emejulu, J.D. (Éd.). 2002: 230262.

Mba-Nkoghe, J. 1991. Le Gabon linguistique. Notre Librairie. Littérature gabonaise 105: 20-23.

Mihindou, G.-R. 2001. Apports des missionnaires à la lexicographie gabonaise: dictionnaires bilingues fang-français/français-fang; français-yipounou/yipounou-français; français/mpongwé. Emejulu J.D. (Éd.). 2001: 7-37.

Nyangone Assam, B. 2001. La lexicographie dans l'apprentissage des langues gabonaises. Emejulu, J.D. (Éd.). 2001: 187-205.

Nyangone Assam, B. et P.A. Mavoungou. 2000. Lexicography in Gabon: A Survey. Lexikos 10: 252274.

Raponda-Walker, A. 1960. Notes d'histoire du Gabon. Montpellier: Imprimerie Charité.

Tomba Moussavou, F. 2002. Quelques aspects de la lexicographie monolingue au Gabon. Emejulu, J.D. (Éd.). 2002: 159-173. 